\title{
BMJ Open The Magnetic Couples Study: protocol for a mixed methods prospective cohort study of HIV-serodifferent heterosexual couples' perspectives and use of pre- exposure prophylaxis (PrEP)
}

To cite: McMahon JM, Simmons J, Haberer JE, et al. The Magnetic Couples Study: protocol for a mixed methods prospective cohort study of HIV-serodifferent heterosexual couples' perspectives and use of pre-exposure prophylaxis (PrEP). BMJ Open 2021;11:e048993. doi:10.1136/ bmjopen-2021-048993

- Prepublication history and additional supplemental material for this paper are available online. To view these files, please visit the journal online. To view these files, please visit the journal online (http://dx.doi org/10.1136/bmjopen-2021048993).

Received 28 January 2021 Accepted 14 June 2021

D Check for updates

(c) Author(s) (or their employer(s)) 2021. Re-use permitted under CC BY-NC. No commercial re-use. See rights and permissions. Published by BMJ.

For numbered affiliations see end of article.

Correspondence to Dr James M. McMahon; james_mcmahon@urmc. rochester.edu

\section{ABSTRACT}

Introduction HIV transmission within serodifferent heterosexual couples plays a key role in sustaining the global HIV pandemic. In the USA, transmission within established mixed-status couples accounts for up to half of all new HIV infections among heterosexuals. Oral HIV preexposure prophylaxis (PrEP) is a highly effective prevention method, although underutilised among serodifferent couples. Moreover, there is a dearth of research on US HIV-serodifferent couples' perspectives and use of PrEP, alone or in combination with other prevention methods. In this paper, we describe the study protocol for the Magnetic Couples Study, designed to fill critical knowledge gaps regarding HIV-serodifferent heterosexual couples' perspectives, experiences and utilisation of PrEP. Methods and analysis The Magnetic Couples Study is a mixed methods prospective cohort study designed to describe temporal patterns and identify determinants at multiple levels (individual, couple, HCF) of PrEP outcomes along the care continuum (PrEP awareness, linkage, uptake, retention and medication adherence) among HIVserodifferent heterosexual couples residing in New York City. The study will also examine clinical management of PrEP, side effects and changes in sexual-related and substance use-related behaviour. A prospective cohort of 230 mixedstatus couples already on oral PrEP was recruited, with quarterly assessments over 18 months; in addition, a crosssectional sample of 150 mixed-status couples not currently on PrEP was recruited. In-depth semistructured qualitative interviews were conducted with a subsample of 25 couples. Actor-partner interdependence modelling using multilevel analysis will be employed for the analysis of longitudinal dyadic data. Framework analysis will be used to analyse qualitative data. A parallel convergent design will be used for mixed methods integration.

Ethics and dissemination The study was approved by the University of Rochester Institutional Review Board (RSRB00052766). Study findings will be disseminated to community members and providers and to researchers and policy makers.
Strengths and limitations of this study

Prospective cohort study of pre-exposure prophylaxis (PrEP) implementation involving HIV-serodifferent heterosexual couples in the USA, a high-risk but understudied population.

- Longitudinal collection of quantitative, qualitative biological and medical record data, including objective measures of PrEP adherence.

- Examination of multilevel (individual, couple, healthcare facility) determinants of PrEP outcomes on the continuum of care.

- Sampling of study participants receiving PrEP and antiretroviral therapy across multiple healthcare facilities allowing for examination of site-level determinants.

- Threats to internal and external validity include bias from self-reported measures, sampling bias and lack of generalisability to non-urban areas.

\section{BACKGROUND}

HIV transmission within heterosexual couples in which one partner is HIV-negative and the other is living with HIV-so-called HIVserodifferent couples-plays a key role in the global HIV pandemic, ${ }^{1-5}$ with couple-linked seroconversions accounting for between $30 \%$ and $50 \%$ of new HIV infections. ${ }^{467}$ In the USA, about one-in-four heterosexuals living with HIV has an established primary sexual partner who is HIV-negative; ${ }^{8-19}$ and such 'mixed-status' or 'magnetic' couples account for up to half of all new HIV infections among heterosexuals nationally. ${ }^{20}$ The risk of HIV transmission within serodifferent couples has been observed to vary widely, from $0 \%$ to $20 \%$ per annum, depending on the type 
and frequency of sexual behaviour and use of prevention methods. ${ }^{21}$

Until relatively recently, condoms were the only effective method of preventing HIV transmission within serodifferent couples. However, despite awareness of their mixed HIV status, consistent condom use among serodifferent couples ranges across studies from a low of $20 \%$ upwards to $75 \% .{ }^{121622-24}$ Moreover, male condoms are only $70 \%-80 \%$ effective at preventing HIV transmission. ${ }^{25-27}$ In contrast, robust evidence has shown that maintaining an undetectable viral load in the partner living with HIV provides complete protection from sexually transmitted HIV in serodifferent couples-affirming the dictum Undetectable $=$ Untransmittable $(\mathrm{U}=\mathrm{U}) \cdot{ }^{28-30}$ Yet, there is limited evidence on the extent to which HIV-serodifferent couples are able to sustain viral suppression; a few studies indicate that only about half of partners living with HIV in mixed-status heterosexual couples are able to maintain long-term viral control (ie, sustained undetectable viral load). ${ }^{23} 31$ Barriers to sustained undetectable viral load include social determinants of health including racism, homelessness, poverty and access to medical care, which can inhibit one's ability to adhere to a daily medication regimen. ${ }^{32-35}$ Oral HIV pre-exposure prophylaxis (PrEP; $200 \mathrm{mg}$ emtricitabine (FTC) in combination with $300 \mathrm{mg}$ tenofovir disoproxil fumarate (TDF) or $25 \mathrm{mg}$ tenofovir alafenamide (TAF)) has been added to the HIV prevention toolbox within the last decade. When taken daily as an oral medication, PrEP has demonstrated high levels of efficacy $(>95 \%)$ in preventing HIV transmission among HIV-serodifferent heterosexual couples. ${ }^{36} 37$

The US Centers for Disease Control and Prevention and the WHO have consequently recognised HIV-serodifferent couples as a high-priority group and have issued recommendations for PrEP and antiretroviral therapy (ART) to prevent dyadic transmission. ${ }^{38-40}$ However, there is a lack of data on PrEP awareness, acceptability, uptake and adherence among HIV-serodifferent heterosexual couples in the USA. ${ }^{2041}$ There is also a dearth of research on couples' perspectives and use of PrEP in combination with ART and other prevention methods, and on the factors at multiple levels (individual, couple, healthcare facilities (HCFs)) that account for enacted prevention strategies in these couples. ${ }^{42-44}$ In addition, there is little evidence on how different HIV prevention strategies might affect sexual and substance use-related behaviours among US mixed-status couples. ${ }^{20}$

The Magnetic Couples Study is an ongoing prospective cohort study funded by the National Institutes of Health, National Institute of Mental Health (R56MH103047; R01MH107371), designed to fill critical knowledge gaps regarding HIV-serodifferent heterosexual couples' perspectives on and experiences in the PrEP care continuum, with the goal of providing an evidence base to inform tailored HIV prevention interventions for this group. The specific aims of the Magnetic Couples Study are as follows: (1) examine trends and identify multilevel (individual, couple, HCF) determinants of awareness, acceptability, uptake and retention of oral PrEP use among HIV-negative partners in US serodifferent heterosexual couples; (2) describe the temporal patterns and identify multilevel determinants of daily oral PrEP and ART adherence in both members of HIV-serodifferent couples, including the pharmacokinetic relationship between self-reported PrEP adherence and FTC/TDF/ TAF drug concentrations in blood; (3) describe the temporal patterns and identify determinants of PrEP care utilisation compliance with recommended HIV testing and clinical management (PrEP visits, HIV testing and ancillary tests, counselling) as well as side effects and toxicity among PrEP users; and (4) identify predictors of changes in sexual and substance use-related behaviours among PrEP users and their partners living with HIV. In the current paper, we describe the study protocol of the Magnetic Couples Study.

\section{METHODS AND ANALYSES \\ Design and samples}

The Magnetic Couples Study employs a mixed methods observational design to characterise trends in PrEP use and identify deterrents and facilitators at multiple levels at each stage of the PrEP care continuum among heterosexual HIV-serodifferent couples. The study does not include provision of clinical care to participants, but examines PrEP utilisation and determinants of care as they occur in real-world settings. The study population consists of mutually disclosed HIV-serodifferent heterosexual couples in which members are age 18 years or older; in a primary heterosexual relationship for at least 3 months and have engaged in vaginal or anal sex in the last 30 days; are fluent in either English or Spanish; and reside in or around the New York City area. The construct of heterosexuality was based on dyadic identity as an opposite-gender/opposite-sex couple and could include both cisgender and transgender persons.

The HIV-serodifferent sample ( $\mathrm{n}=580$; 290 dyads $)$ is composed of two arms: one consisting of a prospective cohort sample ( $\mathrm{n}=460$; 230 dyads), for which the HIVnegative partner had a current prescription for PrEP at the time of baseline enrolment; and the second consists of a cross-sectional sample ( $\mathrm{n}=300 ; 150$ dyads), for which the HIV-negative partner did not have a current PrEP prescription (figure 1). Couples in the non-PrEP arm were allowed to enrol into the PrEP cohort if they later received a PrEP prescription. Thus, the final sample of 290 unique couples include 230 couples enrolled in the prospective PrEP cohort (140 enrolled directly into the PrEP arm plus 90 couples referred from the non-PrEP arm) and 60 couples from the non-PrEP arm not referred to the PrEP cohort. A purposive subsample ( $\mathrm{n}=25$ dyads) drawn from the prospective cohort will be selected to participate in semistructured in-depth qualitative interviews to provide contextual data to aid in the interpretation of quantitative findings. 


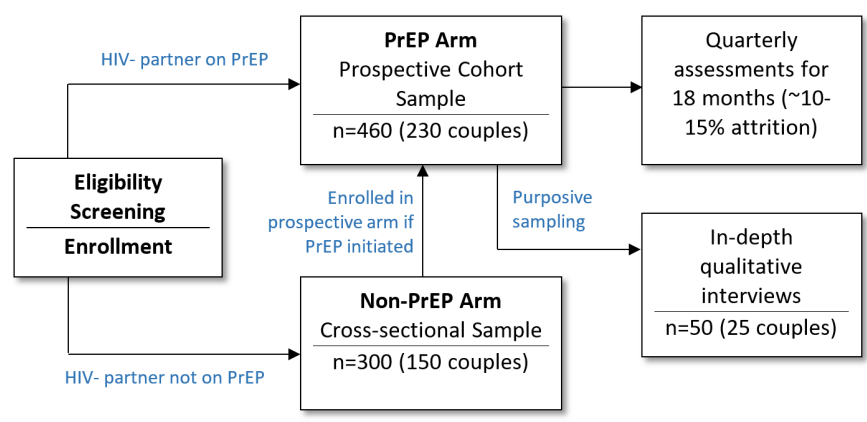

Figure 1 Couple enrolment into pre-exposure prophylaxis (PrEP) and non-PrEP study arms.

\section{Timeline}

Prospective cohort participants are enrolled in the study for an 18-month period, with data collection assessments at intake and at 3-month intervals (maximum seven observations per participant). Both members of each couple provide authorisation to access medical records spanning the period from 18 months prior to study enrolment to their terminal follow-up assessment (total 36 months). Intake enrolment for the study has been completed (June 2016-December 2019) and follow-up assessments are expected to be completed by late 2021 .

\section{Patient and public involvement}

This 5-year PrEP implementation study, supported by the National Institute of Mental Health (NIMH; R01MH107371), was preceded by a National Institutes of Health (NIH) 'High Priority, Short-Term Project Award' (R56MH103047) designed to provide interim support for 'highly meritorious' ... 'creative and innovative approaches' prior to receiving full NIH funding. Conducted from September 2014 to February 2016, our team used this period of support to perform formative work in preparation for the full study, including conducting in-depth interviews with $27 \mathrm{HIV}$-serodifferent couples and 15 PrEP providers. We also interviewed administrators and formed partnerships with over 40 HCFs, service agencies and community-based organisations in New York City. This formative work helped inform all aspects of the study design and data collection. The full study protocol was pilot tested with $10 \mathrm{HIV}$-serodifferent couples prior to enrolling research participants. Public and stakeholder involvement continued during the main study in close consultation and involvement with the New York City PrEP Task Force composed of PrEP providers, patients, advocates, researchers and other stakeholders.

\section{Recruitment and enrolment}

Recruitment efforts focused on increasing awareness of the study among HIV-serodifferent heterosexual couples in the New York City area by employing five primary methods: (1) distribution of study posters and pamphlets (figure 2); (2) passive referrals from clinics, service agencies and community-based organisations; (3) social media campaign (Facebook, Twitter, Craigslist; project website); (4) traditional advertisements in newspapers, magazines,

\section{WE'RE LOOKING FOR PARTICIPANTS!}

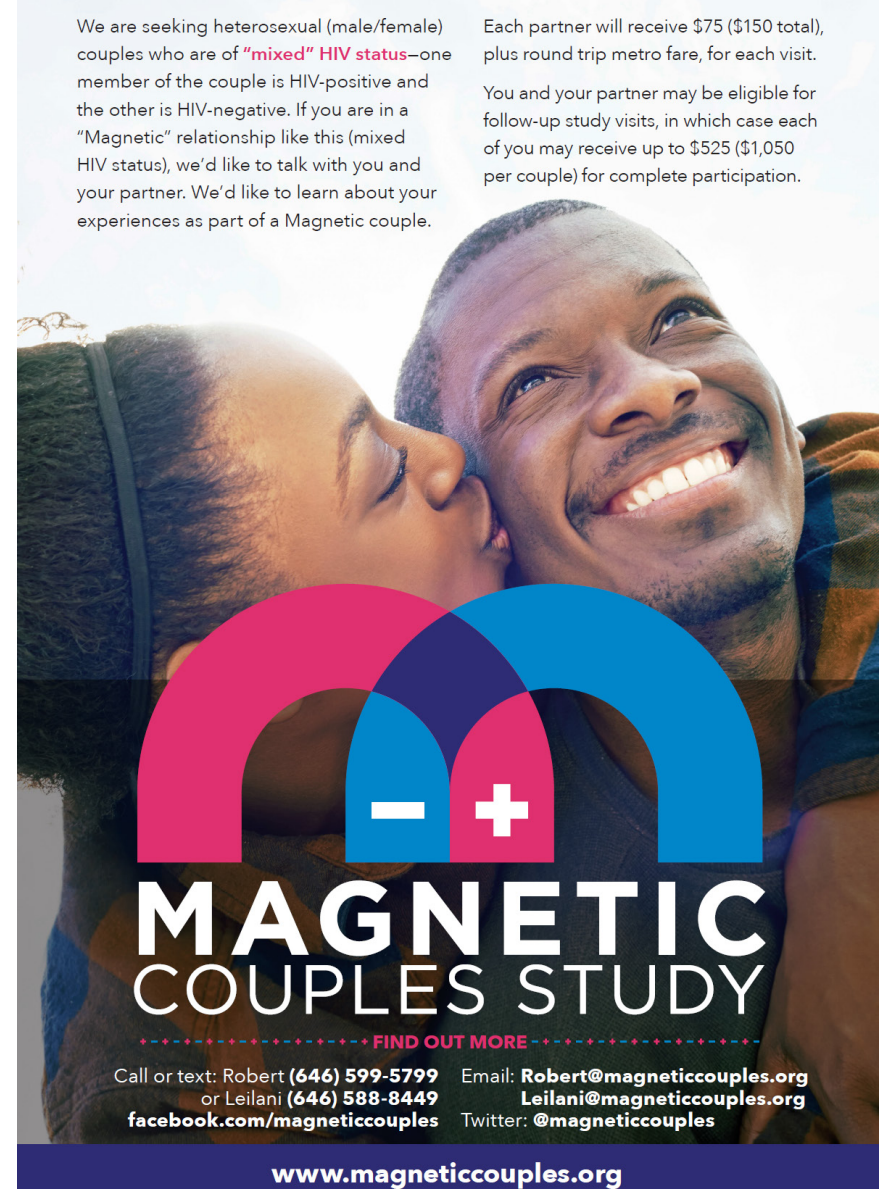

Figure 2 English version poster for the Magnetic Couples Study (Image source: Getty Images \#476852698).

subway signs and radio; and (5) peer-referral with a maximum of three referrals per enrolled couple. These methods directed those interested in the study to contact study staff directly by calling a toll-free phone number, text messaging, emailing or accessing the study website to obtain further information and undergo initial eligibility screening. Those who met the eligibility criteria were offered assistance for informing and recruiting their primary partner into the study using strategies developed by Witte and colleagues. ${ }^{45}$

Once both members of the couple were deemed eligible, an appointment was scheduled for them to visit a centrally located research office in mid-town Manhattan and undergo eligibility verification, informed consent and intake assessment. Eligibility verification consisted of re-screening, HIV testing to confirm HIV-serodifferent status and administration of a couple verification screener to ensure that dyads were primary sexual couples. ${ }^{46} 47$ Eligible couples in which the HIV-negative partner had a current PrEP prescription (verified by the date on their PrEP medication bottle) were enrolled into the prospective cohort PrEP arm of the study; whereas couples not taking PrEP were enrolled into the cross-sectional nonPrEP arm of the study. If couples in the non-PrEP arm 
Table 1 Description of study measures and data collection

\begin{tabular}{|c|c|c|}
\hline Data type & Subjects/Source & Collection method (frequency) \\
\hline Quantitative surveys & $\begin{array}{l}\text { Prospective PrEP arm participants and } \\
\text { cross-sectional non-PrEP participants (each } \\
\text { member of the couple surveyed separately) }\end{array}$ & $\begin{array}{l}\text { Audio computer-assisted self-interviews (ACASI) (PrEP } \\
\text { arm participants: intake, quarterly; non-PrEP arm } \\
\text { participants: intake) }\end{array}$ \\
\hline Medical records & $\begin{array}{l}\text { Prospective PrEP arm participants (HIV- } \\
\text { negative and HIV-positive partners) }\end{array}$ & $\begin{array}{l}\text { Retrospective chart review and electronic extraction } \\
\text { from participating clinics (past } 3 \text { years of records } \\
\text { obtained at terminal follow-up) }\end{array}$ \\
\hline Serum creatinine level & Prospective PrEP arm, HIV-negative partner & $\begin{array}{l}\text { Dried blood spot (DBS) assay for creatinine (intake and } \\
\text { quarterly) }\end{array}$ \\
\hline PrEP drug levels & Prospective PrEP arm, HIV-negative partner & $\begin{array}{l}\text { DBS assay for quantification of TFV-DP (TDF, TAF) and } \\
\text { FTC-TP (intake and quarterly) }\end{array}$ \\
\hline $\begin{array}{l}\text { Healthcare facility } \\
\text { characteristics }\end{array}$ & $\begin{array}{l}\text { Extracted from quantitative surveys; medical } \\
\text { records; public sources }\end{array}$ & Secondary data extraction \\
\hline
\end{tabular}

FTC-TP, emtricitabine triphosphate; PrEP, pre-exposure prophylaxis; TAF, tenofovir alafenamide; TDF, tenofovir disoproxil fumarate; TFV-DP, tenofovir-diphosphate.

expressed an interest in PrEP, a referral was made to a PrEP provider at a preferred location. Couples enrolled in the prospective PrEP cohort who broke up during the study period were allowed to be retained in the study, but follow-up assessments for former partners were scheduled on different days. Survey data were collected on relationship status, break-ups and establishment of new relationships, which may have an impact on PrEP outcomes. Allowing both members of dissolved couples to remain in the study also reduced the risk of potential partner conflict. Contact information was collected and entered into a locator form for all enrolled couples. Each member of the couple is paid US $\$ 75$ for each completed office visit assessment, in addition to a travel reimbursement of US $\$ 5.50$ for subway fares to and from the research office. Enrolled couples are also paid US $\$ 10$ for each couple they refer into the study, with a limit of three referrals per couple.

\section{Measures and data collection}

The Magnetic Couples Study involves the collection of multiple types of data: quantitative surveys, medical record chart review and extraction, biological assays and qualitative interviews (table 1). Consistent with our specific aims, study outcome measures include PrEP acceptability, uptake, and retention (Aim 1), PrEP adherence (Aim 2), utilisation of PrEP clinical care and PrEPrelated side effects (Aim 3), and sexual and substance use-related behaviour change (Aim 4).

\section{Quantitative surveys}

Structured quantitative surveys are administered to all enrolled participants in either English or Spanish by trained bilingual interviewers using a combination of computer-assisted personal interview (CAPI) and audio computer-assisted self-interview (ACASI) methods (online supplemental file 1). Couples are interviewed concurrently but separately in private offices. Members of HIV-serodifferent couples in the prospective PrEP arm complete the survey at baseline and quarterly for 18 months, whereas couples in the non-PrEP cross-sectional arm complete only a single intake survey. Surveys last between 1 and 1.5 hours and are designed with skip patterns such that each survey is tailored to the characteristics of the participant.

Quantitative survey items encompass ten major domains: (1) Demographics (eg, age, race, ethnicity, economic indicators, family composition); (2) General Health (eg, physical and mental health and treatment, history of sexually transmitted infections, reproductive health); (3) Health Systems (eg, provider satisfaction, access and barriers to care, healthcare utilisation); (4) Healthcare Costs (eg, insurance coverage, assistance programmes, out-of-pocket expenses); (5) Structural Factors (eg, stigma, discrimination, incarceration); (6) Psychosocial Factors (eg, social support, resilience); (7) Relationship Function (eg, gender roles, conflict resolution, communication, balance of power, closeness, commitment, trust, intimate partner violence); (8) HIV (eg, ART adherence, disclosure); (9) PrEP (eg, awareness, knowledge, acceptability, utilisation, adherence, formulation preference); (10) HIV Exposure and Prevention (eg, perceived HIV vulnerability, condom attitudes and use, viral suppression, sexual behaviour, substance use behaviour). 


\section{Medical records}

Participants enrolled in the prospective PrEP arm are asked to voluntarily provide Health Insurance Portability and Accountability Act (HIPAA) authorisation to release their medical records obtained from their HCFs delivering ART (for participants living with HIV) or PrEP care (for HIV-negative participants); authorisation forms are completed at intake and updated at terminal follow-up assessment and requests to release medical records are submitted to appropriate HCFs to obtain participant records covering the preceding 36 months. Data obtained from medical records consist of 60 items per office visit and are extracted under the supervision of a nurse study coordinator trained and experienced in medical chart review and extraction. Medical record data cover the following domains: office visit date and purpose; health insurance; vital signs; major diagnoses (physical, mental and sexual health); tests and procedures performed; HIV test results; HIV biometrics (eg, CD4; viral load); ART adherence (provider notes from patient reports or pharmacy refill); ART regimens; PrEP history and care; other prescription medications; kidney and liver function; bone mineral density; vitamin/supplement use; ancillary services; and sexual history.

\section{HIV antibody test}

The HIV-serodifferent status of all couples (PrEP and non-PrEP arms) was confirmed at intake by HIV testing. In addition, all HIV-negative partners are administered an HIV test at each follow-up assessment. HIV testing is performed using the OraQuick Rapid Antibody Test Advance HIV-1/2 kit, with test kit controls performed on new batches (OraSure Technologies). Standard CDC HIV counselling is performed as part of the HIV testing protocol.

\section{Serum creatinine level}

As an indicator of renal function, serum creatinine levels are measured at intake and at all follow-up visits for HIVnegative participants in the PrEP arm. Dried blood spot (DBS) specimens are collected from participants using the PTS Pod System (PTS Diagnostics) and shipped to a commercial laboratory (CoreMedica, Lee's Summit, Missouri, USA) where assays are run and results are reported back via a secure portal. Creatinine levels are then converted to creatinine clearance values applying standard algorithms.

\section{PrEP drug levels}

PrEP (Truvada and Descovy) drug blood levels in HIVnegative participants are quantified as an objective measure of PrEP adherence. Descovy was approved by the US Food and Drug Administration (FDA) for PrEP part-way through data collection and some participants had their prescriptions switched to Descovy by their providers. DBS specimens are collected from HIVnegative partners enrolled in the PrEP arm at intake and quarterly follow-up assessments using the PTS Pod System.
Specimens are shipped to an academic laboratory at the University of Colorado Anschutz Medical Campus for assay, which quantifies (1) tenofovir-diphosphate (TFVDP) concentration (fmol/punch (es)), an estimate of the average amount of PrEP taken over the prior 2-month period; and (2) emtricitabine triphosphate (FTC-TP) concentration (pmol/punch(es)) for which a detectable sample indicates that the participant took at least 1 dose of PrEP in the last 48-72 hours for Truvada and up to the last week for Descovy. ${ }^{48} 49$

\section{Qualitative interviews}

A subsample of HIV-serodifferent couples $(\mathrm{n}=50 ; 25$ dyads) from the prospective PrEP arm participate in face-to-face in-depth semistructured qualitative interviews lasting about 1 hour (online supplemental file 2). Composition of the purposive sample will ensure variation on demographics, PrEP retention and adherence, condom use, and ART adherence and viral load. Once long-acting injectable (LAI) PrEP is approved and available, we will conduct an additional 10-15 interviews with HIV-negative partners who switch to LAI-PrEP. Interviews are conducted concurrently with each member of the couple individually in separate private offices; each partner receives US $\$ 50$ compensation. Interviews cover relationship dynamics, dyadic strategies for HIV prevention, sexual and substance use history, healthcare utilisation, including HIV or PrEP care, and experiences with providers and HCFs. The audio-recorded interviews are transcribed verbatim.

\section{HCF characteristics}

Characteristics of HCFs at which participants receive their PrEP or ART care will be extracted from multiple secondary data sources, including participant quantitative surveys, medical record data and public sources. Characteristics include type (eg, primary care; sexual health clinic), location (eg, access to public transportation), size (patient pool; number of providers), patient resources (eg, onsite pharmacy; social services); and PrEP and ART programmes (eg, support groups; patient navigation; financial assistance).

\section{Data analysis}

\section{Quantitative analysis}

Descriptive statistics will be used to characterise the sample on demographics and key variables. Generalised estimating equation (GEE) analysis will be performed on baseline data pooled from the PrEP and non-PrEP arms to identify predictors of PrEP acceptability and uptake. The actor-partner interdependence modelling (APIM) framework using multilevel modelling (MLM) will be employed for the analysis of longitudinal dyadic data in order to accommodate the nested data structure (repeated observations nested within individuals nested within dyads) and examine mutual partner influence over time in distinguishable dyads. ${ }^{50} 51$ This will permit estimation of PrEP user growth trajectories on outcomes 
while directly assessing the impact of structural, dyadic and partner factors (time-variant and invariant) on outcomes. ${ }^{52}{ }^{53}$ Multilevel models will also be specified to examine the interplay among PrEP adherence, ART adherence/viral suppression and condom use over time. MLM accounts for missing data (under assumptions of missing completely at random or missing at random) by full information maximum likelihood estimation. ${ }^{54}$ Multilevel survival analysis will be used to model time to first event (eg, undetectable TFV-DP level), repeat events (eg, HIV testing) and termination of states (discontinuation of PrEP).$^{55}$ SAS software (V.9.4; SAS Institute, Cary, North Carolina, USA) will be used to perform descriptive, GEE and APIM-MLM analyses; MPlus (V.8.4; Muthen \& Muthen, Los Angeles, California, USA) will be used to perform survival analysis.

\section{Power analysis}

To determine appropriate sample sizes, power calculations were performed using NCSS-PASS software (V.21.0.2) with alpha $=0.05$ and power $=0.80$ for two-sided hypotheses. Based on previous observations, adjustment for within-dyad correlation was set to $\mathrm{r}=0.30$; and for longitudinal analysis, repeated measures correlation was set to $\mathrm{r}=0.50$; we further assumed $15 \%$ attrition at terminal follow-up. For GEE analysis modelling PrEP uptake (binary outcome), a baseline sample of $n=290$ dyads (PrEP and non-PrEP arm observations) can detect an $\mathrm{OR}=1.35$ or higher. For a three-level MLM of continuous outcomes (eg, PrEP medication adherence) and time to event (eg, time to PrEP discontinuation), a sample size of $\mathrm{n}=460$ (230 dyads) can detect an effect size of Beta $=0.30$ $\left(\mathrm{R}^{2}=0.09\right)$.

\section{Qualitative analysis}

Transcribed audio-recorded interviews will be loaded into Dedoose (www.dedoose.com) software for analysis. Following Lewis et $a l l^{57}$ a five-step framework analysis approach will be used to analyse and contextualise the qualitative data. ${ }^{58}$ These steps include the following: (1) Familiarisation: transcripts will be read by the qualitative analysis team and descriptive summaries of each interview will be compiled, from which potential themes will be described; (2) Thematic framework: an initial set of deductive codes will be drawn from our conceptual framework and interview guides and combined with inductive categories drawn from emergent themes identified during familiarisation, producing a nascent code/ subcode list; (3) Indexing: applying the code list, transcripts will be systematically coded with linked excerpts by multiple team members; redundant coding, comparison and iterative code modification with quality checks (eg, inter-rater reliability) will be used to produce a final consensus code list; (4) Charting: at the thematic level, a hierarchy of themes (and cross-linked codes) will be organised by primary outcomes, socioecological level, and content; (5) Mapping and interpretation: charted themes will be summarised.

\section{Mixed methods integration}

For qualitative and quantitative data integration, we will follow the NIH 'Best Practices for Mixed Methods' guidelines established by Creswell and colleagues. ${ }^{59}$ These guidelines provide a framework and taxonomy for the design, integrated analysis and interpretation of qualitative and quantitative data in mixed methods research. Within this framework, we will employ a parallel convergent design, in which qualitative and quantitative data are collected concurrently, with integration at the completion of data collection. Mixed methods data integration will involve connecting, embedding and merging. Qualitative data will be collected from a subsample of the quantitative cohort (connecting) and will be embedded within the larger quantitative study. Data merging will proceed through separate parallel analyses followed by matching on common themes using mixed data matrices structured by level and topic area to provide comparative depth and expansion of understanding. ${ }^{60}$ Triangulation will employ a meta-inference framework, in which the coherence of data integration is interpreted as either confirmation, expansion or discordance. Confirmation occurs when both qualitative and quantitative analyses yield corroborating results; expansion is marked by the two sources of data illuminating different or complementary aspects of the phenomenon; and discordance occurs when qualitative and quantitative findings are inconsistent. ${ }^{61}$ Quantitative analysis will test hypotheses regarding the association of multilevel determinants on PrEP utilisation outcomes, with integrated qualitative data used to confirm, expand or contrast our understanding of how life history, contextual influences and participant perspectives shape these associations.

\section{ETHICS AND DISSEMINATION}

The protocol and procedures for the Magnetic Couples Study have been reviewed and approved by the institutional review board of the University of Rochester (RSRB00052766). The inadvertent loss of confidential and personal information is of primary concern for this study as it could pose potential risks to participants, such as legal consequences or social repercussions. To prevent this from occurring, study staff will maintain strict confidentiality and HIPAA requirements. ACASI methods in which participants directly enter responses to sensitive items into a computer programme help maintain confidentiality. In addition, all participant data are stored in deidentified form, with the exception of a locator form used to schedule follow-up visits, which is stored on a secure server with restricted access. A certificate of confidentiality was issued by the NIH to further protect sensitive information and subject confidentiality.

A second, related, ethical concern pertains to potential partner conflict or violence stemming from couples' joint 
participation in the study. Methods developed by our team to successfully mitigate this concern include (1) being transparent regarding the content and format of the study protocols; (2) allowing each partner to self-select out of the study if they are concerned about their safety; (3) physically separating members of a couple for screening, enrolment and data collection, and instructing them not to discuss any aspects of the study with each other; and (4) providing research staff with training on partner notification programmes for newly HIV-diagnosed partners, and for dealing with and providing referrals for cases involving intimate partner violence. ${ }^{62}$

Future dissemination of study findings will target four primary audiences: community members, providers, researchers and policy makers. Study findings will be shared with community members and providers through the dissemination of infographics through social media and a website as well as by holding forums and presentations in appropriate venues. Researchers and policy makers will be informed of study results primarily through scientific and policy conference presentations and journal articles. Emphasis will be placed on interpretation of findings towards implications for practice and actionable steps.

\section{DISCUSSION}

Although the efficacy of daily oral PrEP for HIV prevention in heterosexual serodifferent couples is well established, implementation research examining determinants of PrEP uptake, retention, adherence and clinical management among US mixed-status heterosexual couples is lacking. ${ }^{20}$ Despite the many ongoing and completed PrEP demonstration and implementation projects, ${ }^{63}$ to our knowledge, the Magnetic Couples Study is the only prospective cohort study to examine PrEP implementation among HIV-serodifferent heterosexual couples in the USA.

In this paper, we describe the study protocol of the Magnetic Couples Study, which was developed through a collaboration of researchers at the University of Rochester, the New York City Department of Health and Mental Hygiene, New York University, Columbia University, Massachusetts General Hospital/Harvard University, and the University of Colorado in partnership with over 40 HCFs, service agencies and community-based organisations in New York City.

Strengths of the study include (1) implementation of a mixed methods multilevel approach involving the collection of quantitative and qualitative data; (2) collection of data on the characteristics of HCFs where participants receive their ART or PrEP care, allowing for analysis of associations with HCF characteristics; (3) extraction of study participants' medical record data from clinical sites to assess medical history, ART and PrEP care, and healthcare utilisation; (4) assays of DBS samples collected from HIV-negative partners at each assessment visit to objectively measure PrEP adherence; (5) HIV-testing of both partners at intake to confirm HIV-serodifferent status, and continued HIV testing of the negative partner to document seroconversions; (6) collection of data from both members of HIV-serodifferent couples, which allows for more accurate modelling of measurement error, dyadic function and mutual influence; (7) 18-month follow-up assessments on quantitative surveys and biological data and 36-month medical record review allowing for analysis of longer-term trends in ART and PrEP utilisation and their determinants; (8) a comparative sample of HIV-serodifferent couples not on PrEP including a subsample of those who subsequently initiate PrEP and enrol in the prospective cohort, thus allowing for modelling of PrEP uptake as well as retention in care and adherence; and (9) a sampling plan designed to reach participants through multiple forms of media as well as via agency and peer referrals, thus not limited to a few clinical sites.

A number of methodological limitations are also inherent in the study. There is a potential for sampling bias favouring couples who are capable of visiting our research office in mid-town Manhattan during regular working hours; although we attempt to accommodate some couples by scheduling assessments after hours or on weekends, the majority of assessments are conducted during regular work hours. The study is also limited to couples residing in the New York City area and findings may not generalise to less urbanised areas or other regions of the USA. However, New York City contains the highest prevalence of persons living with HIV nationally, including heterosexuals in serodifferent relationships. Our study was open to transgender men and women, as long as they reported being in an opposite-gender serodifferent relationship, but the small subsample of transgender persons enrolled in the study might lack adequate power to perform subgroup analysis. In addition, study results may not fully generalise to same-sex couples as PrEP provision among such couples may be affected by a unique set of determinants and relationship dynamics. ${ }^{204-66}$ Much of the quantitative data is based on self-report, which can be subject to various forms of bias, including social desirability and recall bias. ${ }^{67} \mathrm{We}$ attempted to mitigate these sources of bias by employing ACASI methods for sensitive items in the survey, minimising the recall period, collecting identical data from both members of the couple to better model measurement error, and collecting key variables using biological assays and medical chart review.

Knowledge generated from the Magnetic Couples Study will help inform the development of couple-based models of care for dual HIV treatment and prevention involving HIV-serodifferent heterosexual couples. Such couple-based interventions, while not uncommon in African countries, remain relatively untapped in the USA. ${ }^{68}$ Successful provision of PrEP in this group requires an understanding of factors operating concurrently at multiple levels (individual, couple, HCF) that facilitate or impede optimal implementation. The Magnetic Couples Study will identify multilevel time-variant and 
time-invariant determinants of PrEP implementation outcomes among a cohort of HIV-serodifferent heterosexual couples over an 18-month assessment period. Findings will inform optimal methods for healthcare providers across different settings to provide effective models of PrEP and ART care delivery for HIV-serodifferent USA couples, including issues related to ethics and confidentiality surrounding couple-based care.

\section{Author affiliations}

${ }^{1}$ School of Nursing, University of Rochester Medical Center, Rochester, New York, USA

${ }^{2}$ School of Global Public Health, New York University, New York, New York, USA

${ }^{3}$ Center for Global Health, Massachusetts General Hospital, Boston, Massachusetts, USA

${ }^{4}$ Department of Medicine, Harvard Medical School, Boston, Massachusetts, USA

${ }^{5}$ Department of Medicine, New York City Health + Hospitals Harlem, New York, New York, USA

${ }^{6}$ Department of Medicine, Columbia University Medical Center, New York, New York, USA

${ }^{7}$ Department of Sociology, State University of New York at Geneseo, Geneseo, New York, USA

${ }^{8}$ HIV Health and Human Services Planning Council, New York City Department of Health and Mental Hygiene, Queens, New York, USA

${ }^{9}$ College of Health Science and Technology, Rochester Institute of Technology, Rochester, New York, USA

${ }^{10}$ Department of Health and Nutrition Sciences, Brooklyn College, The City University of New York, Brooklyn, New York, USA

${ }^{11}$ School of Nursing, Yale University, Orange, Connecticut, USA

${ }^{12}$ School of Pharmacy and Pharmaceutical Sciences, University of Colorado Anschutz Medical Campus, Aurora, Colorado, USA

Acknowledgements The authors thank the participants of the Magnetic Couples Study. We also thank Adam Lenio for assistance with marketing materials for recruitment and web design; Drs Chen Zhang and Danielle Alcéna-Stiner for work on the study website; the University of Rochester School of Nursing (UR-SON) Centre for Research Support for work on qualitative transcriptions; Deborah Thayer and Michael Fisher of UR-SON Information Systems for web and technical support; Asher Takoda for assistance with R56 qualitative data management; Dr Brent Johnson for quantitative data management consultation; and Marilyn Nickerson and Anne Woodruff for administrative assistance.

Contributors JMM conceived of the study with guidance from JS. The conceptual framework, study design, data collection protocols and analysis plans were developed by JMM, JS, JH, SM, LT, RQ, GH, ERP and AK. Measures were developed by JMM, JS, JH, SM, NT, AB, PLA, AK, MS, JB and EJP. Dried blood spot protocols and assays for PrEP blood level quantification were developed by PLA. JH, SM, NL and AK contributed clinical expertise and JH and SM developed protocols for biometric data collection and procedures and measures for medical record data extraction, in consultation with JMM and JB. JS led the qualitative component of the study and conducted in-depth interviews. Interview guides were designed by JS, NL, NT, AB and JMM, who also performed qualitative data management and quality assurance. LT served as project director and led the design and implementation of participant recruitment and data acquisition procedures, conducted by RQ, GA, AJ, JS, MS and EJP. JMM prepared the initial draft of the manuscript with assistance from GA. All authors critically reviewed and revised manuscript drafts and approved the final version.

Funding The Magnetic Couples Study is funded by the National Institute of Mental Health (Grants R56MH103047 and R01MH107371; James M. McMahon, Principal Investigator), with supplemental funding from the Centre for Research Support, School of Nursing, University of Rochester Medical Centre.

Competing interests PLA has received personal fees and grant funding paid to his institution from Gilead Sciences.

Patient consent for publication Not required.

Provenance and peer review Not commissioned; externally peer reviewed.

Supplemental material This content has been supplied by the author(s). It has not been vetted by BMJ Publishing Group Limited (BMJ) and may not have been peer-reviewed. Any opinions or recommendations discussed are solely those of the author(s) and are not endorsed by BMJ. BMJ disclaims all liability and responsibility arising from any reliance placed on the content. Where the content includes any translated material, BMJ does not warrant the accuracy and reliability of the translations (including but not limited to local regulations, clinical guidelines, terminology, drug names and drug dosages), and is not responsible for any error and/or omissions arising from translation and adaptation or otherwise.

Open access This is an open access article distributed in accordance with the Creative Commons Attribution Non Commercial (CC BY-NC 4.0) license, which permits others to distribute, remix, adapt, build upon this work non-commercially, and license their derivative works on different terms, provided the original work is properly cited, appropriate credit is given, any changes made indicated, and the use is non-commercial. See: http://creativecommons.org/licenses/by-nc/4.0/.

\section{ORCID iDs}

James M. McMahon http://orcid.org/0000-0003-4707-5141

Janie Simmons http://orcid.org/0000-0002-0320-9139

\section{REFERENCES}

1 Muessig KE, Cohen MS. Advances in HIV prevention for serodiscordant couples. Curr HIVIAIDS Rep 2014;11:434-46.

2 Mendelsohn JB, Calzavara L, Daftary A, et al. A scoping review and thematic analysis of social and behavioural research among HIVserodiscordant couples in high-income settings. BMC Public Health 2015;15:241.

3 Sawada I, Tanuma J, Do CD, et al. High proportion of HIV serodiscordance among HIV-affected married couples in northern Vietnam. PLoS One 2015;10:e0125299.

4 Chemaitelly H, Abu-Raddad LJ. Characterizing HIV epidemiology in stable couples in Cambodia, the Dominican Republic, Haiti, and India. Epidemiol Infect 2016;144:90-6.

5 Jia Z, Mao Y, Zhang F, et al. Antiretroviral therapy to prevent HIV transmission in serodiscordant couples in China (2003-11): a national observational cohort study. Lancet 2013;382:1195-203.

6 Li L, Liang L-J, Lin C, et al. Gender differences in depressive symptoms among HIV-positive concordant and discordant heterosexual couples in China. Psychol Women Q 2017;41:89-99.

7 Dunkle KL, Stephenson R, Karita E, et al. New heterosexually transmitted HIV infections in married or cohabiting couples in urban Zambia and Rwanda: an analysis of survey and clinical data. Lancet 2008;371:2183-91.

8 Mizuno Y, Purcell DW, Latka MH, et al. Is sexual serosorting occurring among HIV-positive injection drug users? Comparison between those with HIV-positive partners only, HIV-negative partners only, and those with any partners of unknown status. AIDS Behav 2010;14:92-102.

9 Nacius LA, Levison J, Minard CG, et al. Serodiscordance and disclosure among HIV-positive pregnant women in the Southwestern United States. AIDS Patient Care STDS 2013;27:242-7.

10 Knowlton AR, Yang C, Bohnert A, et al. Main partner factors associated with worse adherence to HAART among women in Baltimore, Maryland: a preliminary study. AIDS Care 2011;23:1102-10.

11 Renaud TC, Bocour A, Tsega A, et al. Do sexual risk behaviors differ between heterosexual youth infected with HIV perinatally versus sexually? J Adolesc Health 2013;53:222-7.

12 Schönnesson LN, Atkinson J, Williams ML, et al. A cluster analysis of drug use and sexual HIV risks and their correlates in a sample of African-American crack cocaine smokers with HIV infection. Drug Alcohol Depend 2008;97:44-53.

13 Spikes PS, Purcell DW, Williams KM, et al. Sexual risk behaviors among HIV-positive black men who have sex with women, with men, or with men and women: implications for intervention development. Am J Public Health 2009;99:1072-8.

14 Starks TJ, Millar BM, Parsons JT. Predictors of condom use with main and casual partners among HIV-positive men over 50. Health Psychol 2015;34:1116-22.

15 Thomson KA, Dhanireddy S, Andrasik M, et al. Fertility desires and preferences for safer conception strategies among people receiving care for HIV at a publicly-funded clinic in Seattle, WA. AIDS Care 2018;30:121-9.

16 Liu C, Hu H, Goparaju L, et al. Sexual serosorting among women with or at risk of HIV infection. AIDS Behav 2011;15:9-15.

17 Braunstein SL, Udeagu C-C, Bocour A, et al. Identifying the correlates of membership in HIV-serodiscordant partnerships in New York City. Sex Transm Dis 2013;40:784-91. 
18 McMahon JM, Braksmajer A, Zhang C, et al. Syndemic factors associated with adherence to antiretroviral therapy among HIVpositive adult heterosexual men. AIDS Res Ther 2019;16:1-10.

19 Farthing $\mathrm{H}$, Rodriguez A, Armstrong M, et al. Discomfort discussing HIV/AIDS and lack of awareness are barriers to partner-topartner pre-exposure prophylaxis education. Int J STD AIDS 2019;30:147-53.

20 McMahon JM, Myers JE, Kurth AE, et al. Oral pre-exposure prophylaxis (PreP) for prevention of HIV in serodiscordant heterosexual couples in the United States: opportunities and challenges. AIDS Patient Care STDS 2014;28:462-74.

21 Lasry A, Sansom SL, Wolitski RJ, et al. HIV sexual transmission risk among serodiscordant couples: assessing the effects of combining prevention strategies. AIDS 2014;28:1521-9.

22 Eaton A, van Der Straten A. Concurrent sexual partnerships among individuals in HIV sero-discordant heterosexual couples. Int J STD AIDS 2009;20:679-82.

23 El-Bassel N, Jemmott JB, Landis JR, et al. National Institute of mental health multisite Eban HIV/STD prevention intervention for African American HIV serodiscordant couples: a cluster randomized trial. Arch Intern Med 2010;170:1594-601.

24 Tripathi A, Whiteside YO, Duffus WA. Perceptions and attitudes about preexposure prophylaxis among seronegative partners and the potential of sexual disinhibition. South Med J 2013;106:558-64.

25 Hughes JP, Baeten JM, Lingappa JR, et al. Determinants of percoital-act HIV-1 infectivity among African HIV-1-serodiscordant couples. J Infect Dis 2012;205:358-65.

26 Weller S, Davis K. Condom effectiveness in reducing heterosexual HIV transmission. Cochrane Database Syst Rev 2002:CD003255.

27 Giannou FK, Tsiara CG, Nikolopoulos GK, et al. Condom effectiveness in reducing heterosexual HIV transmission: a systematic review and meta-analysis of studies on HIV serodiscordant couples. Expert Rev Pharmacoecon Outcomes Res 2016;16:489-99.

28 Cohen MS, Chen YQ, McCauley M, et al. Prevention of HIV1 infection with early antiretroviral therapy. $N$ Engl J Med 2011;365:493-505

29 Loutfy MR, Wu W, Letchumanan M, et al. Systematic review of HIV transmission between heterosexual serodiscordant couples where the HIV-positive partner is fully suppressed on antiretroviral therapy. PLoS One 2013;8:e55747.

30 Rodger A, Cambiano V, Bruun T, et al. Risk of HIV transmission through condomless sex in MSM couples with suppressive art: the PARTNER2 study extended results in gay men. Presented at the 22nd International AIDS Conference, Amsterdam, July 23-27, 2018.

31 Blackstock OJ, Patel VV, Felsen U, et al. Pre-exposure prophylaxis prescribing and retention in care among heterosexual women at a community-based comprehensive sexual health clinic. AIDS Care 2017:29:866-9.

32 Aidala AA, Wilson MG, Shubert V, et al. Housing status, medical care, and health outcomes among people living with HIV/AIDS: a systematic review. Am J Public Health 2016;106:e1-23.

33 Bradley ELP, Frazier EL, Carree T, et al. Psychological and social determinants of health, antiretroviral therapy (art) adherence, and viral suppression among HIV-positive black women in care. AIDS Care 2019;31:932-41

34 Benson C, Wang X, Dunn KJ, et al. Antiretroviral adherence, drug resistance, and the impact of social determinants of health in HIV-1 patients in the US. AIDS Behav 2020;24:3562-73.

35 Rojas D, Melo A, Moise IK, et al. The association between the social determinants of health and HIV control in Miami-Dade County ZIP codes, 2017. J Racial Ethn Health Disparities 2021;8:763-72.

36 Baeten JM, Donnell D, Ndase P, et al. Antiretroviral prophylaxis for HIV prevention in heterosexual men and women. $N$ Engl J Med 2012;367:399-410.

37 Haberer JE, Baeten JM, Campbell J, et al. Adherence to antiretrovira prophylaxis for HIV prevention: a substudy cohort within a clinical trial of serodiscordant couples in East Africa. PLoS Med 2013;10:e1001511

38 World Health Organization. Guidance on oral pre-exposure prophylaxis (PrEP) for serodiscordant couples, men and transgender women who have sex with men at high risk of HIV: recommendations for use in the context of demonstration projects, July 2012, 2012.

39 World Health Organization. Guidance on couples HIV testing and counselling including antiretroviral therapy for treatment and prevention in serodiscordant couples: recommendations for a public health approach, 2012. Available: http://www.who.int/hiv/pub/ guidelines/9789241501972/en/index.html [Accessed 24 Aug 2020].

40 Centers for Disease Control and Prevention. Preexposure prophylaxis for the prevention of HIV infection in the United States - 2014 A Clinical Practice Guideline, 2014: 1-67.
41 Young I, McDaid L. How acceptable are antiretrovirals for the prevention of sexually transmitted HIV?: a review of research on the acceptability of oral pre-exposure prophylaxis and treatment as prevention. AIDS Behav 2014;18:195-216.

42 Hallett TB, Baeten JM, Heffron R, et al. Optimal uses of antiretrovirals for prevention in HIV-1 serodiscordant heterosexual couples in South Africa: a modelling study. PLoS Med 2011;8:e1001123.

43 Falcão JRSP, Bradshaw CC, Garrett CC, et al. Views of HIV-negative partners in heterosexual serodiscordant relationships regarding HIV pre-exposure prophylaxis: a qualitative study. Sex Health 2016;13:345-52

44 Persson A. Notes on the concepts of 'serodiscordance' and 'risk' in couples with mixed HIV status. Glob Public Health 2013;8:209-20.

45 Witte SS, El-Bassel N, Gilbert L, et al. Recruitment of minority women and their main sexual partners in an HIV/STI prevention trial. $J$ Womens Health 2004;13:1137-47.

46 McMahon JM, Tortu S, Torres L, et al. Recruitment of heterosexual couples in public health research: a study protocol. BMC Med Res Methodol 2003;3:24.

47 Syvertsen JL, Robertson AM, Abramovitz D, et al. Study protocol for the recruitment of female sex workers and their non-commercial partners into couple-based HIV research. BMC Public Health 2012;12:136.

48 Anderson PL, Liu AY, Castillo-Mancilla JR, et al. Intracellular tenofovir-diphosphate and emtricitabine-triphosphate in dried blood spots following directly observed therapy. Antimicrob Agents Chemother 2018;62:e01710-7.

49 Yager J, Castillo-Mancilla J, Ibrahim ME, et al. Intracellular tenofovirdiphosphate and emtricitabine-triphosphate in dried blood spots following tenofovir alafenamide: the TAF-DBS study. J Acquir Immune Defic Syndr 2020;84:323-30.

50 Kenny DA, Kashy DA. Dyadic data analysis using multileve modeling.. In: Hox J, Kyle Roberts J, eds. Handbook of advanced multilevel analysis. New York: Taylor \& Francis, 2011: 335-70.

51 Garcia RL, Kenny DA, Ledermann T. Moderation in the actor-partner interdependence model. Pers Relatsh 2015;22:8-29.

52 Livert D, Rindskopf D, Saxe L, et al. Using multilevel modeling in the evaluation of community-based treatment programs. Multivariate Behav Res 2001;36:155-83.

53 Planalp EM, Du H, Braungart-Rieker JM, et al. Growth curve modeling to studying change: a comparison of approaches using longitudinal dyadic data with distinguishable dyads. Struct Equ Modeling 2017;24:129-47.

54 Black AC, Harel O, Betsy McCoach D. Missing data techniques for multilevel data: implications of model misspecification. J App/ Stat 2011;38:1845-65.

55 Yau KKW. Multilevel models for survival analysis with random effects. Biometrics 2001;57:96-102.

56 Singer JD, Willett JB. Applied longitudinal data analysis. modeling change and event occurrences. New York, NY: Oxford University Press, 2003.

57 Lewis MA, Fitzgerald TM, Zulkiewicz B, et al. Identifying synergies in multilevel interventions. Health Educ Behav 2017:44:236-44.

58 Ritchie J, Spencer L. Qualitative data analysis for applied policy research. In: Huberman MA, Miles MB, eds. The qualitative research companion. London: Sage Publications, 2002: 305-29.

59 Creswell JW, Klassen AC, Plano Clark VL. Best practices for mixed methods research in the health sciences. Washington, DC: Office of Behavioral and Social Sciences Research (OBSSR), National Institutes ofHealth (NIH), 2011.

60 Teddie C, Tashakkori A. Foundations of mixed methods research. integrating quantitative and qualitative approaches in the social and behavioral sciences. Thousand Oaks, CA: SAGE, 2009.

61 Fetters MD, Curry LA, Creswell JW. Achieving integration in mixed methods designs-principles and practices. Health Serv Res 2013;48:2134-56.

62 McMahon JM, Chimenti R, Trabold N, et al. Risk of intimate partner violence and relationship conflict following couple-based HIV prevention counseling: results from the Harlem river couples project. $J$ Interpers Violence 2017;32:3709-34.

63 AVAC. Ongoing and planned PrEP demonstration and implementation studies. [Internet], 2019. Available: http:// wwwavacorg/resource/ongoing-and-planned-prep-demonstrationand-implementation-studies [Accessed 29 Aug 2020].

64 Rhodes T, Cusick L. Love and intimacy in relationship risk management: HIV positive people and their sexual partners. Sociol Health IIIn 2000;22:1-26.

65 Remien RH, Dolezal C, Wagner GJ, et al. The association between poor antiretroviral adherence and unsafe sex: differences by gender 
and sexual orientation and implications for scale-up of treatment as prevention. AIDS Behav 2014;18:1541-7.

66 Ko N-Y, Wu H-J, Strong C, et al. Differences in awareness of and willingness to use pre-exposure prophylaxis and anticipated condom use among serodiscordant couples in Taiwan. AIDS Care 2018;30:1306-10.
67 Althubaiti A. Information bias in health research: definition, pitfalls, and adjustment methods. J Multidiscip Healthc 2016;9:211-7.

68 Leblanc NM, McMahon J. Perceived facilitators and barriers to couples' HIV testing and counseling in U.S. clinical settings: perspectives from U.S. health providers. J Assoc Nurses AIDS Care 2019;30:279-91. 